\title{
Implications of pneumonitis after chemoradiation and durvalumab for locally advanced non-small cell lung cancer
}

\author{
Comron Hassanzadeh ${ }^{1}$, Timothy Sita ${ }^{2}$, Rohan Savoor ${ }^{2}$, Pamela P. Samson ${ }^{1}$, Jeffrey Bradley ${ }^{3}$, \\ Michelle Gentile ${ }^{2}$, Michael Roach ${ }^{4}$, Nisha Mohindra ${ }^{5}$, Saiama Waqar ${ }^{6}$, Timothy J. Kruser ${ }^{2}$, \\ Clifford Robinson ${ }^{1}$
}

${ }^{1}$ Department of Radiation Oncology, Washington University School of Medicine, St. Louis, MO, USA; ${ }^{2}$ Department of Radiation Oncology, Northwestern Memorial Hospital, Chicago, IL, USA; ${ }^{3}$ Department of Radiation Oncology, Winship Cancer Institute, Emory University, Atlanta, GA, USA; ${ }^{4}$ Departmen of Radiation Oncology, Cancer Center of Hawaii, Honolulu, HI, USA; ${ }^{5}$ Feinberg School of Medicine, Northwestern University, Chicago, IL, USA; ${ }^{6}$ Department of Medicine, Oncology Division, Washington University School of Medicine, St. Louis, MO, USA

Contributions: (I) Conception and design: C Robinson, C Hassanzadeh, M Roach, TJ Kruser, T Sita; (II) Administrative support: C Robinson, C Hassanzadeh, TJ Kruser, T Sita; (III) Provision of study materials or patients: C Robinson, C Hassanzadeh, TJ Kruser, T Sita; (IV) Collection and assembly of data: C Robinson, C Hassanzadeh, TJ Kruser, T Sita; (V) Data analysis and interpretation: C Robinson, C Hassanzadeh, TJ Kruser, T Sita; (VI) Manuscript writing: All authors; (VII) Final approval of manuscript: All authors.

Correspondence to: Clifford Robinson, MD. Department of Radiation Oncology, Washington University School of Medicine, 4921 Parkview Place, Campus Box 8224, St. Louis, MO 63110, USA. Email: clifford.robinson@wustl.edu.

Background: Consolidation durvalumab improved overall survival (OS) in locally advanced non-small cell lung cancer (LA-NSCLC) treated with chemoradiotherapy (CRT) in the PACIFIC trial; however, pneumonitis was increased with durvalumab. We sought to examine real-world outcomes with the PACIFIC paradigm, especially factors associated with pneumonitis, using a multi-institutional review.

Methods: Patients with LA-NSCLC treated with CRT followed by durvalumab from January 2017February 2019 were identified at 2 institutions. We characterized demographics, tumor factors, radiotherapy, and duration of durvalumab. We examined pneumonitis outcomes including re-challenge success, with secondary endpoints of progression-free survival (PFS) and OS.

Results: Thirty-four patients were included with median follow-up of 12 months (range, 3 to 20 months); $94 \%$ had stage III disease. The cumulative grade $>2$ pneumonitis rate was $26.5 \%$ with 2 patients developing grade 3 pneumonitis and no grade $4 / 5$ events. Median time to pneumonitis after RT was 2.4 months (range, 0 to 4.9 months). Pneumonitis management included median prednisone dose of $60 \mathrm{mg}$ for median taper of 6 weeks with durvalumab held for median of 4.5 weeks (range, 2 to 8 weeks); $70 \%$ of pneumonitis patients received durvalumab re-challenge, with pneumonitis recurring in $14 \%$ of patients. 3 -month and 6-month pneumonitis-free-survival were $76.9 \%$ and $73.6 \%$, respectively; 9- and 12 -month OS were $96 \%$ (75.1-99.8\%), 86.6\% (63.5-95.5\%), respectively; 9- and 12-month PFS were 68\% (47.5-82.5\%), 48.7\% (25.3-68.3\%). Pneumonitis development did not significantly impact PFS or OS $(\mathrm{P}>0.05)$.

Conclusions: Among LA-NSCLC patients treated with CRT followed by consolidation durvalumab, more than $25 \%$ developed symptomatic pneumonitis. In this small case series, pneumonitis did not appear to negatively impact survival, and durvalumab re-challenge appeared feasible after pneumonitis treatment with steroids.

Keywords: Non-small cell lung cancer (NSCLC); durvalumab; chemoradiotherapy (CRT); pneumonitis; immune-related adverse events (irAE)

Submitted Apr 29, 2020. Accepted for publication Sep 09, 2020.

doi: $10.21037 /$ jtd-20-1792

View this article at: http://dx.doi.org/10.21037/jtd-20-1792 


\section{Introduction}

Non-small cell lung cancer (NSCLC) remains the leading cause of cancer-related mortality in the US with more than $30 \%$ of patients diagnosed with locally advanced disease (1-3). The current standard of care for patients with inoperable stage III NSCLC and a good performance status is concurrent chemoradiotherapy (CRT) using platinum-based doublet chemotherapy with the recent addition of consolidation immunotherapy with the immune checkpoint inhibitor (ICI) durvalumab (4-7). The addition of consolidation immunotherapy is due to the recent results of PACIFIC, a phase 3 randomized trial comparing consolidation durvalumab versus placebo in patients with unresectable stage III NSCLC who had not progressed after definitive concurrent chemoradiation and demonstrated significantly improved progression-free (PFS) and overall survival (OS) (4).

While ICIs have prolonged survival for patients with locally advanced NSCLC (LA-NSCLC), oncologists must be aware of the potential for severe or life-threatening immune-mediated adverse events, especially pneumonitis (8). PACIFIC reported rates of pneumonitis of any grade for durvalumab and placebo of $33.9 \%$ and $24.8 \%$, respectively, and grade 3 and 4 pneumonitis rates of $3.4 \%$ and $2.6 \%$, respectively. Pneumonitis was the most frequent adverse event leading to the discontinuation of durvalumab and led to discontinued treatment in $6.3 \%$ of patients (4). In addition, pneumonitis can limit quality of life, lead to oxygen dependence, and limit future therapeutic interventions $(9,10)$.

Radiation pneumonitis is among the most clinically challenging toxicities following lung radiation and may be exacerbated by consolidation immunotherapy. This potential intensification of toxicity was highlighted in the KEYNOTE-001 trial which used the checkpoint inhibitor pembrolizumab for advanced NSCLC and observed pulmonary toxicity of any grade in $63 \%$ of patients with prior thoracic radiation versus $40 \%$ of patients without thoracic radiation (11).

Our goals were to perform an analysis of real-world rates of radiation pneumonitis in patients receiving definitive chemoradiation and consolidation durvalumab for the treatment of LA-NSCLC. Furthermore, we sought to describe the impact of pneumonitis on durvalumab dose intensity, and the safety of durvalumab re-challenge after pneumonitis in the setting of definitive chemoradiation. We present the following article in accordance with the
STROBE reporting checklist (available at http://dx.doi. org/10.21037/jtd-20-1792).

\section{Methods}

\section{Study population}

The study was conducted in accordance with the Declaration of Helsinki (as revised in 2013).

After institutional review board of Washington University in St. Louis HRPO\# 201902048 and legal approval, a multi-institution collaboration was established. Individual consent for this retrospective analysis was waived. Data was hosted and analyzed using a central repository maintained with REDCap Software V7.3.5 (Vanderbilt University, Nashville, Tennessee) (12).

Between January 2017 to February 2019, 84 patients with LA-NSCLC treated with concurrent chemoradiation were identified from both institutions. Patients were queried from January 2017 as this was near the time of the adoption of consolidation durvalumab in clinical practice. Patients with stage IIB-IIIC NSCLC were included. Patients were excluded if they received consolidative chemotherapy in lieu of durvalumab, progressed during RT or soon after RT, did not complete the full CRT course, had a pre-existing autoimmune disorder that did not allow for durvalumab administration, underwent prior thoracic RT, or underwent neoadjuvant immunotherapy. The remaining cohort of patients received consolidative durvalumab after definitive CRT for LA-NSCLC. Ultimately, 50 patients were excluded from the analysis (Figure 1).

\section{Radiotherapy}

RT was delivered using intensity modulated RT (IMRT) technique for a planned course of 6-7 weeks of RT. All patients were staged with computerized tomography (CT) of the chest, positron emission tomography (PET), and/or mediastinal staging with endobronchial ultrasound (EBUS) or mediastinoscopy for equivocal PET findings. Patients were followed with serial CT imaging at 6 weeks postRT, then typically every 3 months for 2 years, and every 6 months for an additional 3 years.

RT was delivered using IMRT technique for a planned course of six to seven weeks of RT. Radiation simulation and planning were performed using free-breathing and fourdimensional CT to assess tumor respiratory motion. Gross tumor volumes (GTV) were contoured according to PET/ 


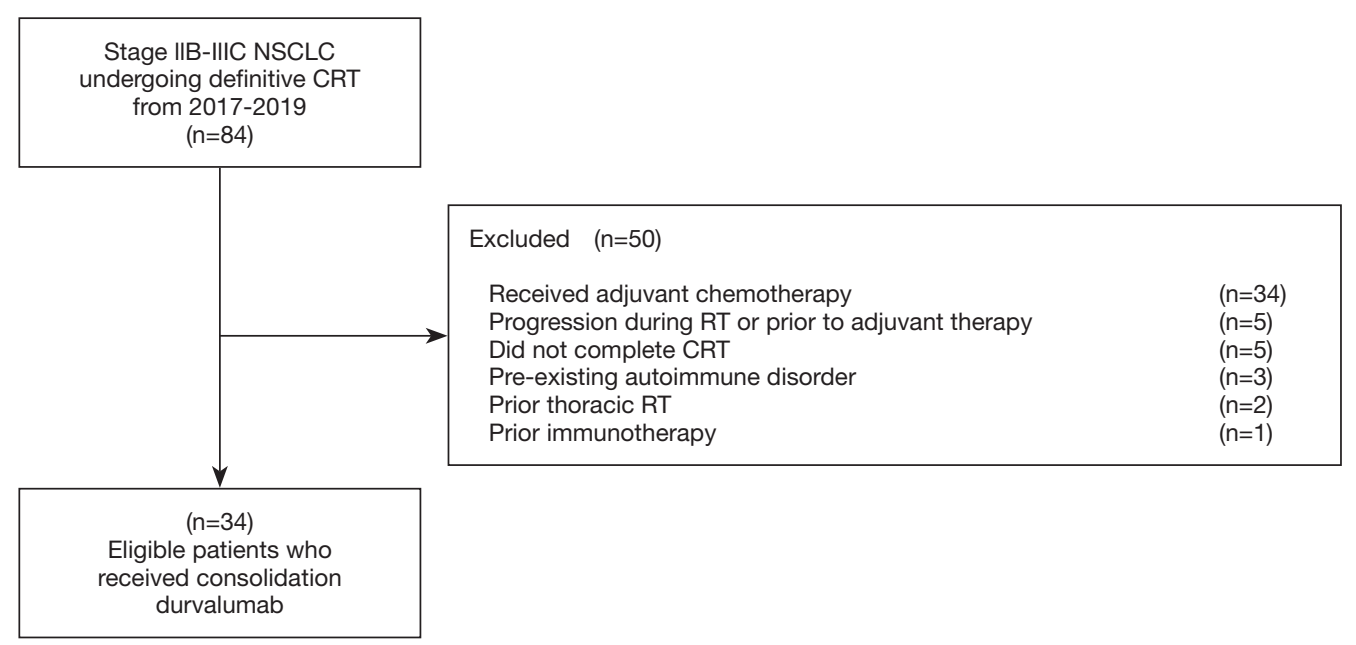

Figure 1 CONSORT diagram of study population. NSCLC, non-small cell lung cancer; CRT, concurrent chemoradiation; RT, radiotherapy.

CT avid primary tumor and nodal involvement. Internal target volumes (ITV) were contoured accounting for respiratory motion of GTV with a 5 to $7 \mathrm{~mm}$ expansion to clinical target volume (CTV) followed by $5 \mathrm{~mm}$ expansion to planning target volume (PTV). Dose constraints to organs-at-risk (OARs) included spinal cord maximum dose of less than 45 Gy, total lung mean dose less than 20 Gy, and heart V30 less than 50\% and heart V50 less than $25 \%$, brachial plexus maximum dose less than $66 \mathrm{~Gy}$, and esophagus mean dose less than 34 Gy. Lung dose-volume histogram parameters were calculated as total lung volumes subtracted from CTV (total lung-CTV) volumes, and are referred to as lung V5, lung V20 with the utilization of dose constraints including lung V20 less than $37 \%$ and V5 less than $60 \%$.

\section{Clinical endpoints}

Patient, tumor, and treatment factors were investigated and compiled into a de-identified database. The following data was collected: age at diagnosis, cancer diagnosis and staging, smoking status, patient demographics, concurrent chemotherapy, concurrent radiation modality, details of concurrent radiation including dose and fractionation, durvalumab course including number of treatments, and radiation dose parameters to the OARs. Patient comorbidities were assessed using the Charlson comorbidity index, adapted by Deyo et al. (13). Physician-reported acute and late toxicity was queried and classified using the Common Terminology Criteria for Adverse Events (CTCAE) scoring system, version 5.0, using the following: patient follow-up notes; CT, PET/CT, or magnetic resonance imaging (MRI); endoscopy; echocardiography; and cardiac catheterization.

Specifically, pneumonitis was determined using documented clinical data regarding patient status, available CT chest imaging, and physician assessment of pneumonitis development given the constellation of findings. Physician reporting of immune-related adverse events (irAE) were also documented according to chart review. These irAE's were attributed to durvalumab when all other probable causes were excluded.

\section{Statistical analysis}

Follow-up time was calculated from the end of radiation therapy (RT). The primary endpoint of the study was pneumonitis development and pneumonitis free survival (time from the end of RT until pneumonitis development, death or censoring at the date of last clinical followup). Pneumonitis that occurred during RT was coded as 0 months or considered to have occurred at the 0 -time point. Secondary endpoints were OS, PFS, and distant metastasis free survival (DMFS). OS was determined as the time from end of RT until death or date of last followup. PFS was determined as the time from end of RT until first progression, death or date of last follow up. DMFS was determined as the time from end of RT until first 
Table 1 Baseline characteristics

\begin{tabular}{|c|c|}
\hline Characteristics & All patients $(\mathrm{N}=34)$ \\
\hline Follow-up, median [range], months & 12 [3-20] \\
\hline \multicolumn{2}{|l|}{ Age, year } \\
\hline Median [range] & 68 [37-84] \\
\hline \multicolumn{2}{|l|}{ Sex, No. (\%) } \\
\hline Male & $21(53.8)$ \\
\hline Female & $13(33.3)$ \\
\hline \multicolumn{2}{|l|}{ Race, No. (\%) } \\
\hline White & $24(61.5)$ \\
\hline Black or African-American & $8(20.5)$ \\
\hline Asian & $1(2.6)$ \\
\hline Unknown & $1(2.6)$ \\
\hline \multicolumn{2}{|l|}{ Stage, No. (\%) } \\
\hline IIIA & $20(51.3)$ \\
\hline IIIB & $11(28.2)$ \\
\hline IIIC & $1(2.6)$ \\
\hline Other $^{\dagger}$ & $2(5.1)$ \\
\hline \multicolumn{2}{|l|}{ ECOG Performance Status Score (\%) } \\
\hline 0 & $10(25.6)$ \\
\hline 1 & $19(48.7)$ \\
\hline 2 & $5(12.8)$ \\
\hline \multicolumn{2}{|l|}{ Histology, No. (\%) } \\
\hline Adenocarcinoma & $15(38.5)$ \\
\hline Squamous & $12(30.8)$ \\
\hline Non-small cell, NOS & $7(17.9)$ \\
\hline \multicolumn{2}{|l|}{ Smoking status, No. (\%) } \\
\hline Current smoker & 7 (17.9) \\
\hline Former smoker & $25(64.1)$ \\
\hline Never smoker & $2(5.1)$ \\
\hline \multicolumn{2}{|l|}{ Charlson/Deyo comorbidity score (\%) } \\
\hline 0 & $7(20.6 \%)$ \\
\hline 1 & $9(26.5 \%)$ \\
\hline 2 & $6(17.6 \%)$ \\
\hline$\geq 3$ & $12(35.3 \%)$ \\
\hline \multicolumn{2}{|l|}{ Concurrent chemotherapy, No. (\%) } \\
\hline Carboplatin/paclitaxel & $30(76.9)$ \\
\hline
\end{tabular}

Table 1 (continued)
Table 1 (continued)

\begin{tabular}{|c|c|}
\hline Characteristics & All patients $(\mathrm{N}=34)$ \\
\hline Cisplatin/etoposide & $3(7.7)$ \\
\hline Other $^{\ddagger}$ & $1(2.6)$ \\
\hline \multicolumn{2}{|c|}{ Radiation dose/fractionation, Gy/fractions } \\
\hline Median (range) & $60[60-70] / 30$ [30-35] \\
\hline \multicolumn{2}{|c|}{ Radiation modality, No. (\%) } \\
\hline IMRT & $30(76.9 \%)$ \\
\hline Protons & $4(10.3 \%)$ \\
\hline \multicolumn{2}{|c|}{$\begin{array}{l}+ \text {, 'other' stage included } 1 \text { patient with stage IIB and } 1 \text { patient } \\
\text { with recurrent stage IIIB; }{ }^{\ddagger} \text {, 'other' concurrent chemotherapy } \\
\text { included } 1 \text { patient receiving carboplatin/etoposide, percentages } \\
\text { may not total } 100 \text { because of rounding. ECOG, Eastern } \\
\text { Cooperative Oncology Group; NOS, not otherwise specified. }\end{array}$} \\
\hline
\end{tabular}

distant metastasis, death, or date of last follow up. KaplanMeier method was used for these endpoints. Potential factors associated with development of pneumonitis were investigated with univariate Cox proportional hazards modeling. Subsequently, all variables with a $\mathrm{P}$ value less than 0.05 or less were entered into the multivariable analysis. All statistical tests were two-sided. A P value less than 0.05 was considered statistically significant. All analysis was completed using SPSS software, version 22.

\section{Results}

\section{Patient outcomes}

A total of 34 patients treated from 2017 to 2019 for clinical stage IIB to IIIC NSCLC treated with definitive chemoradiation followed by consolidation durvalumab were identified using the aforementioned selection criteria (Table 1). Median follow up was 12 months (range, 3-20 months). Median age was 68 (range, 37 to 84 years) with 21 males $(61.7 \%)$ with 10 patients $(29.4 \%)$ with ECOG 0 performance status, 19 patients $(55.8 \%)$ with ECOG 1, and the remaining 5 patients (14.7\%) with ECOG 2 .

The clinical staging for the patient cohort was $59 \%$ with stage IIIA, 32\% with stage IIIB, 1 patient with stage IIIC, 1 patient with recurrent stage IIIA disease, and 1 patient with stage IIB. The median radiation dose and fractionation was 60 Gy (range, 60-70 Gy) in 30 fractions (range, 30-35 fractions) and was delivered over a median of 43 days (range, 
38-51 days).

The median lung V5 and V20 were $48.5 \%$ (range, 19.3$65.0 \%$ ) and $19.4 \%$ (range, $6.7-38.1 \%$ ) and the median lung mean dose was 11.6 Gy (range, 4.4-19.1 Gy). The median esophagus mean dose was 22.9 Gy (range, 5.5-40.7 Gy) and median esophagus V60 was $4.8 \%$ (range, $0-45.4 \%$ ). Median heart mean dose was 10.1 Gy (range, 0.4-43.0 Gy) and median heart V50 was 41.8\% (range, 12.8-53.2\%).

\section{Chemotherapy and immunotherapy}

Definitive chemotherapy regimens included carboplatin/ paclitaxel $(88.2 \%)$, cisplatin/etoposide $(8.8 \%)$ and carboplatin/etoposide (3\%). One patient received neoadjuvant chemotherapy with cisplatin/permetrexed. A median number of 6 doses of chemotherapy were administered during RT. Durvalumab dose was administered at a standard dose of $10 \mathrm{mg} / \mathrm{kg}$ intravenously with no dose reductions for up to 12 months as tolerated. Median number of durvalumab doses administered were 8.5 (range, 1-26 doses). The median time between completion of radiation and start of durvalumab was 29 days (range, 18-128 days).

\section{Impact and management of pneumonitis and irAE}

Median time to pneumonitis from the end of RT was 2.4 months (range, 0-4.9 months). There were 9 patients $(26.5 \%)$ with grade $>2$ pneumonitis among the entire cohort. Seven patients $(20.6 \%)$ developed grade 2 pneumonitis and 2 patients (5.9\%) developed grade 3 pneumonitis. No patients developed grade 4 or 5 pneumonitis. Pneumonitis-free survival for 3 - and 6 -month timepoints was $76.9 \%$ and $73.6 \%$. Pneumonitis development did not significantly impact PFS (70\% with pneumonitis vs. $49.2 \%$ without pneumonitis at 12 months, $\mathrm{P}=0.91)$ or $\mathrm{OS}(100 \%$ vs. $82.4 \%$ at 12 months, $\mathrm{P}=0.31)$.

All patients who developed pneumonitis were prescribed prednisone for symptom management at a median initial dose of $60 \mathrm{mg}$ (range, $40-60 \mathrm{mg}$ ) for a median taper length of 6 weeks (range, 2-19 weeks). Initial prednisone dosing was prescribed by medical oncologists in $89 \%$ of patients with pneumonitis, and by the inpatient medical team in $11 \%$ of patients. Durvalumab was held during the initial steroid course for a median of 4.5 weeks (range, 2-8 weeks). After the initial steroid taper, 7 out of the 9 patients $(77.87 \%)$ with pneumonitis were re-challenged with durvalumab with 1 out of the 7 patients $(14.3 \%)$ developing pneumonitis recurrence after durvalumab rechallenge.

Other physician-reported irAEs during patient's durvalumab course were noted in 7 patients $(20.6 \%)$ including autoimmune thyroiditis in 2 patients $(5.9 \%)$, immune-related diarrhea requiring prednisone taper in 1 patient (3\%), vitiligo development requiring discontinuation of durvalumab in 1 patient (3\%), perforated duodenal ulcer requiring surgical repair in 1 patient $(3 \%)$ and immunerelated transaminitis requiring prednisone taper in 1 patient (3\%). Overall, 9 patients $(26.5 \%)$ required discontinuation of durvalumab due to any toxicity. Among these 9 patients, 6 patients discontinued for pneumonitis, 1 patient for immune-related transaminitis, 1 patient for immune-related diarrhea, and 1 patient for vitiligo development.

\section{Survival outcomes}

Median OS was not reached, while 9-, 12- and 18-month OS rates were $96 \%(75.1-99.8 \%), 86.6 \%$ (63.5-95.5\%) and $74.6 \%(48.5-88.8 \%)$, respectively. Median PFS was 11.8 months (95\% CI, 7.2-16.4 months), while 9-, 12-, and 18 -month PFS were 68\% (47.5-82.5\%), 48.7\% (25.3$68.3 \%$ ), and $38.9 \%$ (15.5-61.3\%), respectively. Median DMFS was 11.9 months (95\% CI, 11.1-16.7 months), while 9- and 12-month DMFS were $72 \%(51-85 \%)$ and $46.7 \%$ (23.7-66.7\%), respectively.

On univariate analysis for OS, there was no association between age, race, smoking status, stage, histology, number of durvalumab cycles administered, and comorbidity score $(\mathrm{P}>0.05)$. On univariate analysis for $\mathrm{PFS}$, patient age $(\mathrm{P}=0.01)$, number of durvalumab cycles administered $(\mathrm{P}=0.002)$ and the Charlson/Deyo comorbidity score $(\mathrm{P}=0.006)$ were associated with worse PFS. Durvalumab cycles administered $(\mathrm{P}=0.003)$ and comorbidity score $(\mathrm{P}=0.008)$ were also significantly associated with PFS on multivariate analysis. On univariate analysis for pneumonitis free survival, only lung V5 ( $\mathrm{P}=0.03)$ was significantly associated with pneumonitis free survival (Figures 2,3, Table 2).

\section{Discussion}

Using a multi-institutional review of patients with LANSCLC, we investigated the real-world management of patients receiving consolidative durvalumab in a curative setting and reported the success of durvalumab re-challenge after pneumonitis development. The PACIFIC trial provides little clarity in real-time management of treatment- 

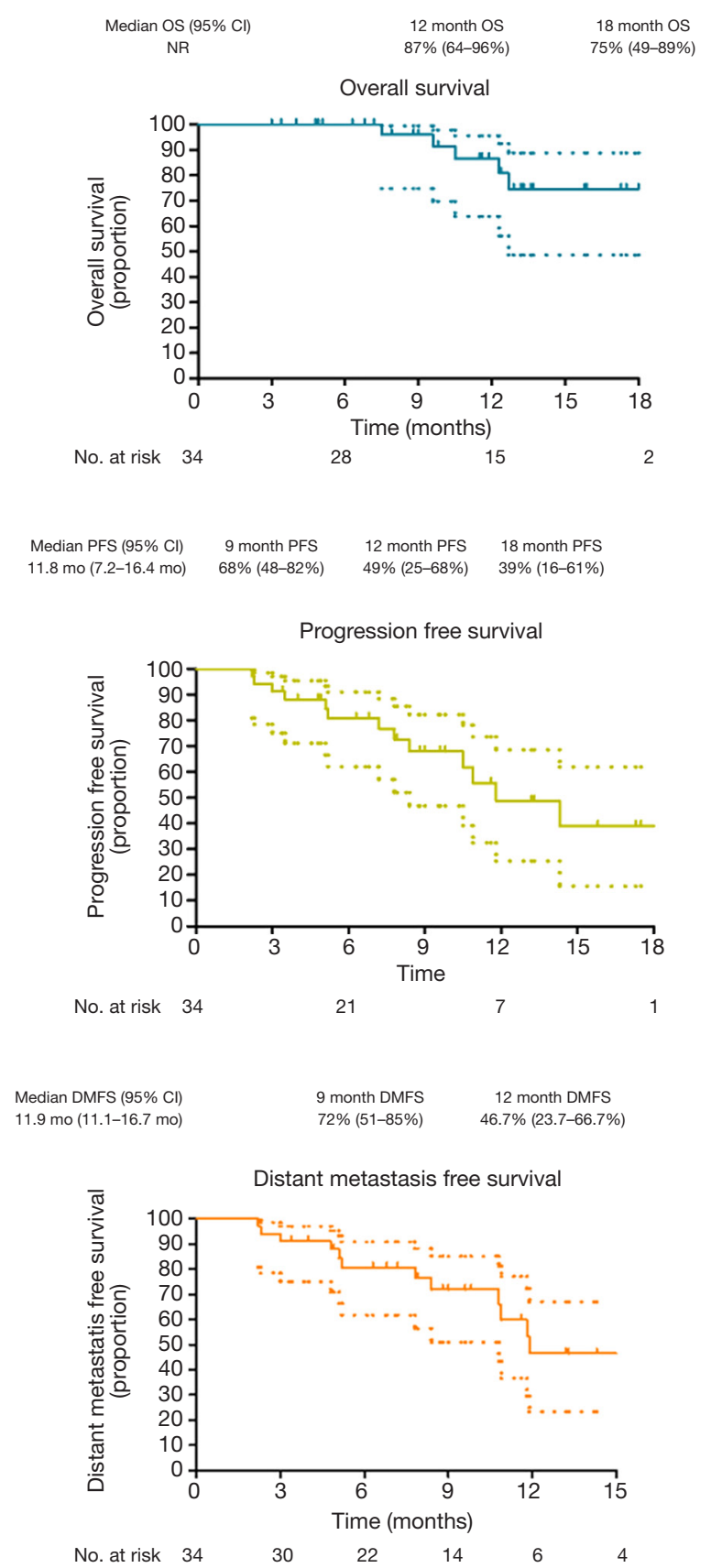

Figure 2 Overall survival, progression-free survival, and distant metastasis-free survival. Shown as Kaplan-Meier curves. Tick marks indicate censored observations. OS, overall survival; PFS, progression-free survival; DMFS, distant metastasis-free survival; CI, confidence interval; NR, not reached.

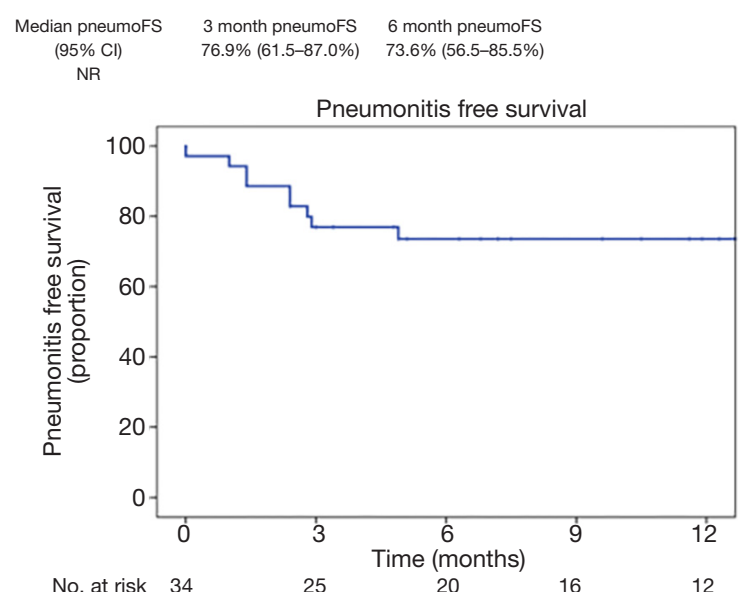

Figure 3 Pneumonitis-free survival. Shown as Kaplan-Meier curves. Tick marks indicate censored observations. PneumoFS, pneumonitis-free survival; CI, confidence interval; NR, not reached.

related pneumonitis and our findings provide insight into overseeing these challenging clinical dilemmas outside of a clinical trial setting (4). Our results demonstrated more than one-quarter of patients developed radiation pneumonitis after definitive CRT and consolidation durvalumab. Pneumonitis development did not appear to be associated with survival outcomes and only 2 patients (5.9\%) developed grade 3 pneumonitis, with no patients developing severe grade $4 / 5$ pneumonitis. In those patients who developed pneumonitis, re-challenge with durvalumab was well tolerated after resolution of symptoms in appropriately selected patients.

Our cumulative rate of grades 2-4 pneumonitis was $26.5 \%$ which is slightly higher than the previously published incidence of pneumonitis with durvalumab consolidation. However, this comparison to the randomized data is limited due to the smaller cohort in our study population. In the PACIFIC study, rates of grades 2-4 pneumonitis were 19\% in the durvalumab arm versus $12 \%$ in the placebo arm (4). PACIFIC reported both pneumonitis and radiation pneumonitis together since these entities are hard to differentiate, with a combined pneumonitis (grades 1-5) rate of $33.9 \%$ in the durvalumab arm compared to $24.8 \%$ in the placebo arm. This is in contrast to the most recent 
Table 2 Univariate and multivariate analyses of factors associated with overall survival, progression-free survival, and pneumonitis-free survival

\begin{tabular}{|c|c|c|c|c|}
\hline Variables & \multicolumn{2}{|c|}{ UVA } & \multicolumn{2}{|c|}{ MVA } \\
\hline \multicolumn{5}{|l|}{ Overall survival and factors associated } \\
\hline Age, years & $0.963(0.89-1.04)$ & 0.34 & & \\
\hline \multicolumn{5}{|l|}{ Race } \\
\hline Black or African-American & $2.44(0.27-22.2)$ & 0.43 & & \\
\hline Other & 0 & 0.99 & & \\
\hline \multicolumn{5}{|l|}{ Smoking status } \\
\hline Current smoker & Ref & & & \\
\hline \multicolumn{5}{|l|}{ Stage } \\
\hline IIIA & Ref & & & \\
\hline IIIB & $0.92(0.15-5.63)$ & 0.93 & & \\
\hline Other & 0 & 0.99 & & \\
\hline \multicolumn{5}{|l|}{ Histology } \\
\hline Adenocarcinoma & Ref & & & \\
\hline Squamous & $2.04(0.13-32.9)$ & 0.62 & & \\
\hline Non-small cell, NOS & $4.45(0.46-42.9)$ & 0.2 & & \\
\hline Charlson/Deyo Comorbidity Score & $0.88(0.58-1.33)$ & 0.54 & & \\
\hline \multicolumn{5}{|c|}{ Progression-free survival and factors associated } \\
\hline Age, years & $0.932(0.88-0.98)$ & $0.011^{\star \star}$ & NS & \\
\hline \multicolumn{5}{|l|}{ Race } \\
\hline White & Ref & & & \\
\hline Black or African-American & $0.49(0.06-4.00)$ & 0.51 & & \\
\hline Other & $0.98(0.12-7.78)$ & 0.98 & & \\
\hline \multicolumn{5}{|l|}{ Smoking status } \\
\hline Current smoker & Ref & & & \\
\hline Former smoker & $0.34(0.07-1.70)$ & 0.19 & & \\
\hline Never smoker & $0.54(0.07-4.08)$ & 0.55 & & \\
\hline
\end{tabular}

Table 2 (continued) 
Table 2 (continued)

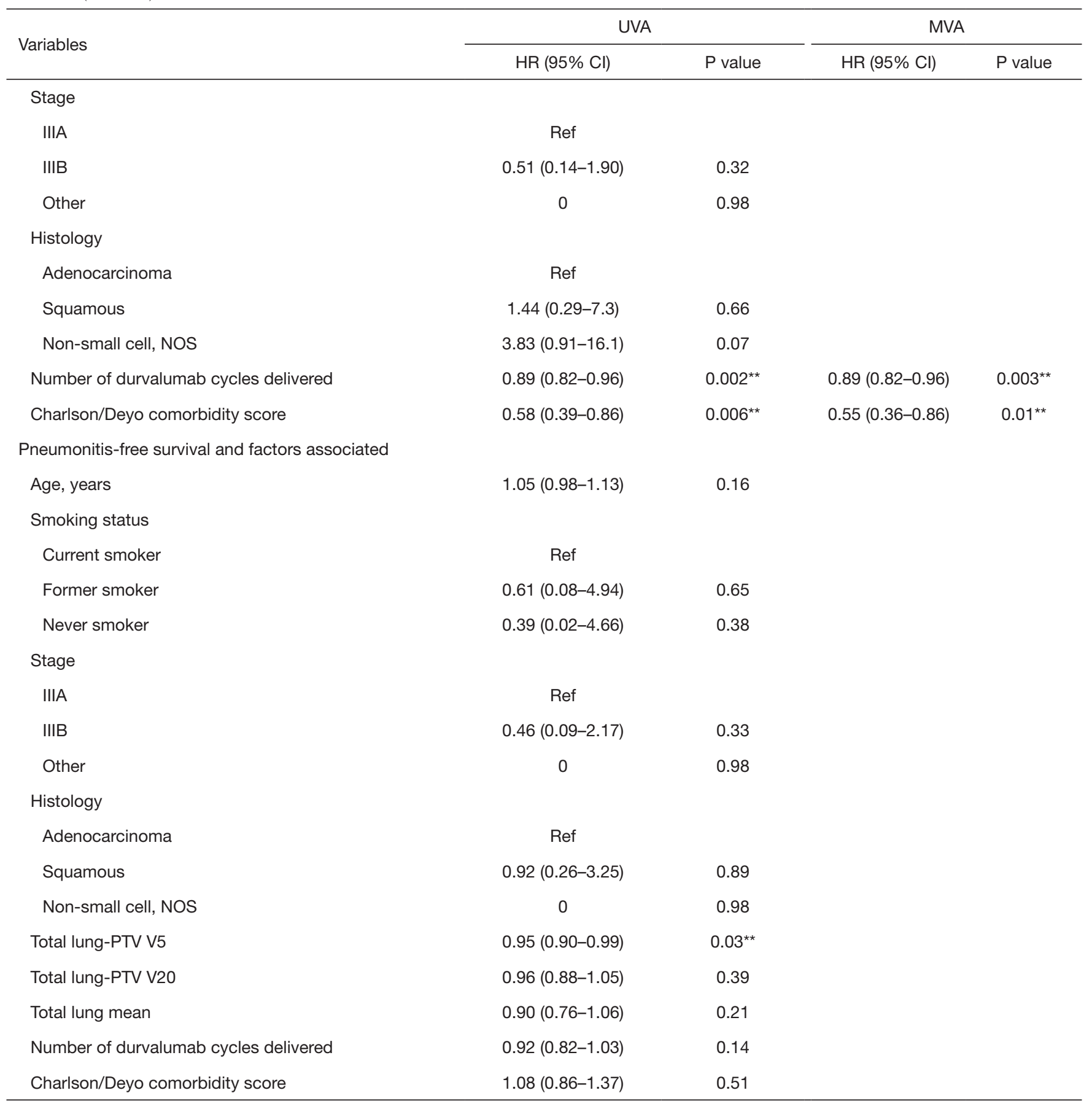

No factors were associated with overall survival. The number of durvalumab cycles delivered and comorbidity score were significantly associated with progression free survival on multivariate analysis. Lung V5 was significantly associated with pneumonitis on univariate. ${ }^{* *}$ denotes statistical significance. UVA, univariate; MVA, multivariate; OS, overall survival; PFS, progression free survival; NOS, not otherwise specified; NS, not significant; HR, hazard ratio; $\mathrm{Cl}$, confidence interval. 
pre-durvalumab era randomized clinical trial, RTOG 0617 where the rate of acute grades $2-4$ pneumonitis was $9-10 \%$ and the rate of late grades $2-4$ pneumonitis was $9 \%$ in the standard dose arm (14). In addition to the synergy between immunotherapy and radiation leading to pneumonitis, chemotherapy may further potentiate this effect. In our data set, $88 \%$ of patients received carboplatin/ paclitaxel chemoradiation. Carboplatin with taxane-based chemotherapy has been identified as a risk factor for pneumonitis development in patients receiving definitive CRT for LA-NSCLC $(9,15)$. However, there was no association identified between chemotherapy choice and pneumonitis development in our cohort.

Current guidelines regarding the safety of re-challenging with ICIs after pneumonitis are sparse and usually favor holding or discontinuing immunotherapy in lieu of rechallenging after resolution of symptoms (16-18). However, this approach may unnecessarily restrict patient access to therapy that may provide life-prolonging benefit. In our cohort, re-challenge with durvalumab in patients who previously discontinued durvalumab due to pneumonitis was well tolerated with only 1 out of 7 patients developing pneumonitis recurrence. While this is a small number to draw strong conclusions from, our results appear to contrast previously reported rates of pneumonitis and irAE recurrence after re-challenge where $26 \%$ of patients had recurrence of the initial irAE and $26 \%$ developed a new irAE after re-challenge (19). Overall, the rate of high dose glucocorticoid usage for our patient set was $26.5 \%$, while PACIFIC reported high dose glucocorticoid use of $8.8 \%$ (4). The median time durvalumab was held was 4.5 weeks with a median initial prednisone dose of $60 \mathrm{mg}$ for a median taper length of 6 weeks. This suggests that durvalumab may not need to be held indefinitely in the event of pneumonitis. Clinical practice guidelines for management of irAEs from ICIs suggest re-challenging patients after proper management of their irAE while cautioning of the risk of irAE recurrence (20).

Our observed survival outcomes are comparable with that of PACIFIC. Our 12 -month OS rate was $86.6 \%$ while PACIFIC demonstrated a 12 -month OS of $83.1 \%$. Our median PFS was 11.8 months while PACIFIC had a median PFS from randomization of 17.2 months. However, our median DMFS rate of 11.9 months was shorter than the reported median time to death or distant metastasis of 28.3 months in PACIFIC (21). This difference in DMFS could be explained by differences in patient clinical factors between our two cohorts and our smaller study cohort.

In this current data set we reviewed the impact of multiple variables on PFS, OS and pneumonitis free survival. Interestingly, the development of pneumonitis did not appear to be negatively associated with worse PFS or OS outcomes. This is in contrast to a study by Suresh et al which found an increased risk of death in patients with NSCLC who developed checkpoint inhibitor associated pneumonitis (22). The number of durvalumab cycles administered and the pre-existing comorbidity score of patients were associated with PFS on multivariate analyses. While the number of durvalumab cycles and development of pneumonitis did not appear to be associated with OS in this series, given the association with PFS of durvalumab and the potential for false negative findings in this small series with limited power to detect survival impacts, these results warrant confirmation in larger examinations. Pneumonitis free survival was associated with lung V5 while lung V20 and lung mean dose were not significantly associated with pneumonitis. In addition, although V20 and mean lung dose are frequently used in clinical practice as dose-volume parameters to reduce the risk of pneumonitis, other dosimetric variables including V5 have also been shown to be predictive as was shown in our study (10). Low dose radiation exposure as represented by V5 may be more important in pneumonitis risk when consolidation immune checkpoint inhibitors (ICIs) are administered (23). Additional investigation into dosimetric parameters and their association with pneumonitis using a larger patient dataset is necessary, especially when considering the synergistic effects of radiation and ICIs contributing to pneumonitis.

The limitations of this study include its retrospective nature, and its small sample size. The conclusions drawn from association of factors and outcomes with pneumonitis may be limited due to this sample size. In addition, adequate data was not available for some variables of interest including number of pack-year smoking history, baseline pulmonary function, and dosimetric data beyond previously reported dose-volume lung parameters for pneumonitis. However, the median follow-up time of our data set was 11 months, whereas the median time to pneumonitis was nearly 2 months, with all pneumonitis events occurring by 6 months. A larger body of retrospective data pooled from multiple institutions in the future will be essential to further understand the impact of durvalumab and other immunotherapy on the development of pneumonitis after CRT. 


\section{Acknowledgments}

Funding: This work was supported by Clinical and Translational Science Award (CTSA) Grant (UL1 TR000448) and Siteman Comprehensive Cancer Center and NCI Cancer Center Support Grant P30 (CA091842).

\section{Footnote}

Reporting Checklist: The authors have completed the STROBE reporting checklist. Available at http://dx.doi. org/10.21037/jtd-20-1792

Data Sharing Statement: Available at http://dx.doi. org/10.21037/jtd-20-1792

Conflicts of Interest: All authors have completed the ICMJE uniform disclosure form (available at http:// dx.doi.org/10.21037/jtd-20-1792). Dr. SW reports grants from 1 UM1 CA186704-01, other from F. HoffmannLa Roche Ltd, other from Ariad, other from Pfizer Pharmaceuticals, Inc., other from Hengrui Therapeutics, other from Xcovery, other from EMD Serono Research \& Development Institute, Inc., other from Checkpoint Therapeutics, Inc., other from Genentech, Inc., other from Lilly, other from Stemcentrx, Inc., other from Ignyta, Inc., other from Bristol-Myers Squibb Pharmaceutical, other from Synermore Biologics Co., Ltd., other from Novartis Pharmaceuticals Corporation, other from Merck \& Company, Inc., other from NewLink Genetics Corporation, other from Celegene, outside the submitted work. Dr. NM reports personal fees from Astra Zeneca, personal fees from Bristol Meyers Squibb, personal fees from Abbvie, personal fees from Genentech, outside the submitted work. Dr. JB reports personal fees from AstraZeneca, outside the submitted work. Dr. TJK reports personal fees from AstraZeneca, personal fees from AstraZeneca, personal fees from Varian Medical Systems, personal fees from Abbvie Inc, outside the submitted work. Dr. MR reports personal fees and non-financial support from ViewRay, non-financial support from Varian, outside the submitted work. Dr. CR reports grants and personal fees from Varian, grants from Elekta, other from Radialogica, outside the submitted work. The other authors have no conflicts of interest to declare.

Etbical Statement: All authors are accountable for all aspects of the work in ensuring that questions related to the accuracy or integrity of any part of the work are appropriately investigated and resolved. The study was conducted in accordance with the Declaration of Helsinki (as revised in 2013). The study was approved by institutional review board of Washington University in St. Louis HRPO\# 201902048 and individual consent for this retrospective analysis was waived.

Open Access Statement: This is an Open Access article distributed in accordance with the Creative Commons Attribution-NonCommercial-NoDerivs 4.0 International License (CC BY-NC-ND 4.0), which permits the noncommercial replication and distribution of the article with the strict proviso that no changes or edits are made and the original work is properly cited (including links to both the formal publication through the relevant DOI and the license). See: https://creativecommons.org/licenses/by-nc-nd/4.0/.

\section{References}

1. McMillan MT, Ojerholm E, Verma V, et al. Radiation Treatment Time and Overall Survival in Locally Advanced Non-small Cell Lung Cancer. Int J Radiat Oncol Biol Phys 2017;98:1142-52.

2. Meza R, Meernik C, Jeon J, et al. Lung Cancer Incidence Trends by Gender, Race and Histology in the United States, 1973-2010. PLoS One 2015;10:e0121323.

3. Cancer statistics, 2017 - Siegel - 2017 - CA: A Cancer Journal for Clinicians - Wiley Online Library [Internet]. [cited 2019 Aug 7]. Available online: https://onlinelibrary. wiley.com/doi/full/10.3322/caac.21387

4. Antonia SJ, Villegas A, Daniel D, et al. Durvalumab after Chemoradiotherapy in Stage III Non-Small-Cell Lung Cancer. N Engl J Med 2017;377:1919-29.

5. Bezjak A, Temin S, Franklin G, et al. Definitive and Adjuvant Radiotherapy in Locally Advanced Non-SmallCell Lung Cancer: American Society of Clinical Oncology Clinical Practice Guideline Endorsement of the American Society for Radiation Oncology Evidence-Based Clinical Practice Guideline. J Clin Oncol 2015;33:2100-5.

6. Bradley J, Graham MV, Winter K, et al. Toxicity and outcome results of RTOG 9311: A phase I-II doseescalation study using three-dimensional conformal radiotherapy in patients with inoperable non-smallcell lung carcinoma. Int J Radiat Oncol Biol Phys 2005;61:318-28.

7. Postmus PE, Kerr KM, Oudkerk M, et al. Early and locally advanced non-small-cell lung cancer (NSCLC): ESMO Clinical Practice Guidelines for diagnosis, treatment and 
follow-up. Ann Oncol 2017;28:iv1-21.

8. Davies M, Duffield EA. Safety of checkpoint inhibitors for cancer treatment: strategies for patient monitoring and management of immune-mediated adverse events. ImmunoTargets Ther 2017;6:51-71.

9. Palma DA, Senan S, Tsujino K, et al. Predicting Radiation Pneumonitis After Chemoradiation Therapy for Lung Cancer: An International Individual Patient Data Metaanalysis. Int J Radiat Oncol Biol Phys 2013;85:444-50.

10. Rodrigues G, Lock M, D'Souza D, et al. Prediction of radiation pneumonitis by dose-volume histogram parameters in lung cancer-a systematic review. Radiother Oncol 2004;71:127-38.

11. Shaverdian N, Lisberg AE, Bornazyan K, et al. Previous radiotherapy and the clinical activity and toxicity of pembrolizumab in the treatment of non-small-cell lung cancer: a secondary analysis of the KEYNOTE-001 phase 1 trial. Lancet Oncol 2017;18:895-903.

12. Harris PA, Taylor R, Thielke R, et al. Research electronic data capture (REDCap) - A metadata-driven methodology and workflow process for providing translational research informatics support. J Biomed Inform 2009;42:377-81.

13. Deyo RA, Cherkin, D, Ciol, M. Adapting a Clinical Comorbidity Index for use with ICD-9-CM administrative database. J Clin Epidemiol 1992;45:613-9.

14. Bradley JD, Paulus R, Komaki R, et al. Standard-dose versus high-dose conformal radiotherapy with concurrent and consolidation carboplatin plus paclitaxel with or without cetuximab for patients with stage IIIA or IIIB non-smallcell lung cancer (RTOG 0617): a randomised, two-by-two factorial phase 3 study. Lancet Oncol 2015;16:187-99.

15. Parashar B, Edwards A, Mehta R, et al. Chemotherapy Significantly Increases the Risk of Radiation Pneumonitis

Cite this article as: Hassanzadeh C, Sita T, Savoor R, Samson PP, Bradley J, Gentile M, Roach M, Mohindra N, Waqar S, Kruser TJ, Robinson C. Implications of pneumonitis after chemoradiation and durvalumab for locally advanced nonsmall cell lung cancer. J Thorac Dis 2020;12(11):6690-6700. doi: $10.21037 /$ jtd-20-1792 in Radiation Therapy of Advanced Lung Cancer. Am J Clin Oncol 2011;34:160-4.

16. Champiat S, Lambotte O, Barreau E, et al. Management of immune checkpoint blockade dysimmune toxicities: a collaborative position paper. Ann Oncol 2016;27:559-74.

17. Weber JS, Yang JC, Atkins MB, et al. Toxicities of Immunotherapy for the Practitioner. J Clin Oncol 2015;33:2092-9.

18. Michot JM, Bigenwald C, Champiat S, et al. Immunerelated adverse events with immune checkpoint blockade: a comprehensive review. Eur J Cancer 2016;54:139-48.

19. Santini FC, Rizvi H, Plodkowski AJ, et al. Safety and Efficacy of Re-treating with Immunotherapy after Immune-Related Adverse Events in Patients with NSCLC. Cancer Immunol Res 2018;6:1093-9.

20. Brahmer JR, Lacchetti C, Schneider BJ, et al. Management of Immune-Related Adverse Events in Patients Treated With Immune Checkpoint Inhibitor Therapy: American Society of Clinical Oncology Clinical Practice Guideline. J Clin Oncol 2018;36:1714-68.

21. Antonia SJ, Villegas A, Daniel D, et al. Overall Survival with Durvalumab after Chemoradiotherapy in Stage III NSCLC. N Engl J Med 2018;379:2342-50.

22. Suresh K, Psoter KJ, Voong KR, et al. Impact of Checkpoint Inhibitor Pneumonitis on Survival in NSCLC Patients Receiving Immune Checkpoint Immunotherapy. J Thorac Oncol 2019;14:494-502.

23. Schoenfeld JD, Nishino M, Severgnini $M$, et al. Pneumonitis resulting from radiation and immune checkpoint blockade illustrates characteristic clinical, radiologic and circulating biomarker features. J Immunother Cancer 2019;7:112. 